\title{
THE HOFFMAN-ROSSI THEOREM FOR OPERATOR ALGEBRAS
}

\author{
DAVID P. BLECHER, LUIS C. FLORES, AND BEATE G. ZIMMER
}

\begin{abstract}
We study possible noncommutative (operator algebra) variants of the classical Hoffman-Rossi theorem from the theory of function algebras. In particular we give a condition on the range of a contractive weak* continuous homomorphism defined on an operator algebra $A$, which is necessary and sufficient (in the setting we explain) for a positive weak* continuous extension to any von Neumann algebra containing $A$.
\end{abstract}

\section{INTRODUCTION}

The Hoffman-Rossi theorem that we are interested in here is a remarkable result from the theory of function algebras [9, Theorem 3.2]. It states that if $A$ is a weak* closed unital subalgebra of $M=L^{\infty}(\mu)$, for a probability measure $\mu$, and if $\varphi$ is a weak* continuous character (i.e. nontrivial complex-valued homomorphism) on $A$, then $\varphi$ has a weak* continuous positive linear extension to $M$. Since unital linear functionals on $M$ (or on any $C^{*}$-algebra) are states (that is, contractive and unital linear functionals) if and only if they are positive, this is saying that weak* continuous characters on $A$ have weak* continuous Hahn-Banach extensions to $L^{\infty}(\mu)$. Or, in the language of von Neumann algebras (see e.g. p. 245, 248-249 in [1]), weak* continuous characters on $A$ have normal state extensions to $L^{\infty}(\mu)$. In the present paper we study possible noncommutative (operator algebra) variants of this result. An operator algebra is a unital algebra of operators on a Hilbert space, or more abstractly a Banach algebra isometrically isomorphic to such an algebra of Hilbert space operators. (Sometimes one wants to consider an operator space structure on an operator algebra, and replace the word 'isometrically' by 'completely isometrically' in the last sentence (see e.g. [5] for definitions), but this will not be important in the present paper.) Our main result is a condition on the range of a contractive (or completely contractive) weak* continuous homomorphism $\Phi$ defined on a unital operator algebra $A$, which is necessary and sufficient in the setting explained below, for a positive weak* continuous extension of $\Phi$ to any von Neumann algebra containing $A$ as a weak* closed subalgebra.

Date: May 21, 2019

1991 Mathematics Subject Classification. Primary 47A64, 47L10, 47L30, 47B44; Secondary 15A24, 15A60, 47A12, 47A60, 47A63, 49M15, 65F30.

Key words and phrases. Operator algebra, noncommutative function theory, extension of linear map, injective von Neumann algebra, conditional expectation.

DB is supported by a Simons Foundation Collaboration Grant. 


\section{A Von Neumann Algebra VAlued Hoffman-Rossi theorem}

There are several natural ways to try to generalize the Hoffman-Rossi theorem to the operator algebra setting. First however we note that the setting of algebras and algebra homomorphisms is crucial. Even in the classical setting, one cannot hope that weak ${ }^{*}$ continuous states on operator systems have weak* continuous HahnBanach extensions (that is, normal state extensions). The following, which we will use later, is a convincing illustration of this.

Example 2.1. Consider the state $\varphi_{1}$ of evaluation at 1 on the set $\mathcal{S}$ of polynomials of degree $\leq 1$ on $[0,1]$, viewed as a subspace of $L^{\infty}([0,1])$. This is weak ${ }^{*}$ continuous (since $\mathcal{S}$ is finite dimensional). Any normal state extension to $L^{\infty}([0,1])$ of $\varphi_{1}$ is integration against a positive $g \in L^{1}([0,1])$ with $\int_{[0,1]} g d t=1$. Applying this state to the monomial $t$ gives $\int_{[0,1]} t g(t) d t=\varphi_{1}(t)=1$. Hence $\int_{[0,1]}(1-t) g(t) d t=0$, forcing the contradiction $g=0$ a.e..

With a little more work one can find a weak* continuous state on a unital weak* closed subalgebra of a von Neumann algebra $M$, which has no normal state extension to $M$ (for example the algebra in the proof of Proposition 2.6 below).

In the noncommutative (operator algebra) setting we suppose that we have a weak* closed unital subalgebra $A$ of a von Neumann algebra $M$, and a weak* continuous unital contractive homomorphism $\Phi: A \rightarrow D$, for a von Neumann algebra $D$. By the first (resp. second) 'unital' here we mean that $1 \in A$, where 1 is the identity of $M$ (resp. $\Phi(1)=1)$. The question we are interested in is when does $\Phi$ have a weak* continuous contractive (or equivalently, positive) $D$-valued linear extension to $M$ ? Sometimes we will add the adjective 'completely', for example consider UCP (unital completely positive) extensions-see e.g. 5, Chapter 1] for notation. To obtain such a Hoffman-Rossi theorem one has to have restrictions on the algebras or on $\Phi$ or its range of $\Phi$, as we will see below. It is not true in general, for example, when $D=B(H)$ for a Hilbert space $H$ (unless $A$ is also selfadjoint, in which case it may be proved using as one ingredient the well known extendibility of normal representations of von Neumann algebras).

In the case of the original Hoffman-Rossi theorem we may identify the range of the character with $D=\mathbb{C} 1_{A}=\mathbb{C} 1_{M}$, in which case the homomorphism we are extending is an idempotent map on $A$, and is a $D$-bimodule map. Thus we will usually restrict our attention to the setting of a weak* continuous unital contractive or completely contractive homomorphism $\Phi: A \rightarrow D \subset A$ which is a $D$-bimodule map (or equivalently, is the identity map on $D$ ). Such maps $\Phi$ are called $D$-characters in a sequel paper [4, where their theory is developed much more extensively. We remark that by 4.2.9 in [5], any unital completely contractive projection of a unital operator algebra onto a subalgebra $D$ is a $D$-bimodule map. We then ask for a positive normal extension from $M$ into $D$. Note that the latter implies that $D$ is a von Neumann algebra, and $\Psi$ is a conditional expectation onto $D$ (see p. $132-133$ in 1] for the main facts about conditional expectations and their relation to bimodule maps and projections maps of norm 1). However $D$ being a von Neumann algebra is not enough for a weak* continuous positive extension from $M$ into $D$, even if $D$ is also injective and commutative. For example, Takesaki [12] showed that there need not exist a positive weak* continuous extension from $M$ onto such $D$ if $M$ is not 'finite'. 
The correct Hoffman-Rossi theorem in the setting described in the last paragraph that works for all von Neumann algebras $M$ requires $D$ to be finite dimensional, or more generally, a (purely) atomic von Neumann algebra (see p. 354 in [1]). This is Theorem 2.4, and the result after it (Proposition 2.5) shows the necessity of the atomic hypothesis.

First however we give a quick proof of the case of our main result in the scalar valued case. This particular proof we noticed some time after our paper was submitted (our original proof is now Remark 2.3). It follows a method which seems to be attributed by Hoffman and Rossi to Heinz König and Don Sarason below Theorem 3.3 in 9. These authors of course addressed the commutative case. A variant of this argument may be found in a (much later) method of Cassier to prove a similar result for characters on singly generated commutative dual operator algebras [6]. These ideas are somewhat buried in [9] and [6]. Indeed the proof of the HoffmanRossi result (stated in our very first paragraph) which is officially presented in 9] and which found its way into the function algebra texts (such as [8]) is several orders of magnitude more complicated. We combine and modify these ideas hidden in [9, 6], so as to include both the noncommutative case and (a generalization of) the original Hoffman-Rossi theorem. Of course the statement in the commutative case is formally contained in the noncommutative result. We have chosen to write it as follows to make the importance of König and Sarason's contribution clearer. In the description of their contribution below Theorem 3.3 in [9], $d \mu=r d m$ in our notation below; $m$ is a positive measure such that $L^{1}(m)^{*}=L^{\infty}(m)$.

Theorem 2.2. (Hoffman-Rossi, the scalar valued case) Let $M=B(H)$ for $a$ Hilbert space $H$ or let $M=L^{\infty}(m)$ be a commutative von Neumann algebra. Suppose that $\varphi: A \rightarrow \mathbb{C}$ is a weak* continuous character on a weak ${ }^{*}$ closed subalgebra $A$ of $M$ containing the unit of $M$. Then $\varphi$ has a normal state extension to $M$.

Proof. To combine the classical and noncommutative case we write $L^{\infty}$ for $B(H)$ or $L^{\infty}(m), L^{1}$ for the predual of $L^{\infty}$, and $\operatorname{tr}$ for the trace on $B(H)$ or for the integral on $L^{\infty}(m)$ (classical case). We also write $L^{2}$ for the Hilbert space with inner product $\operatorname{tr}\left(b^{*} a\right)$ (this will be the Hilbert-Schmidt operators in the $B(H)$ case or $L^{2}(m)$ in the commutative case). By Banach space duality there exists $r \in L^{1}$ such that $\operatorname{tr}(x r)=\varphi(x)$ for $x \in A$. Write $r=a b$ for $a, b \in L^{2}$. Let $J=\operatorname{Ker} \varphi$, and let $E$ (resp. $F$ ) be the closure in the $L^{2}$ norm of $A a$ (resp. $J a$ ). For $f \in J$ we have

$$
\|a-f a\|_{2}^{2}=\operatorname{tr}\left(a^{*}|1-f|^{2} a\right)=\operatorname{tr}\left(|(1-f) a|^{2}\right) \geq \frac{1}{C^{2}}
$$

for a constant $C>0$, since

$$
1=\varphi(1-f)=\operatorname{tr}((1-f) r)=\operatorname{tr}(b(1-f) a) \leq C \operatorname{tr}\left(|(1-f) a|^{2}\right)^{\frac{1}{2}} .
$$

It follows that $a \notin F$, so $E \neq F$. Choosing a unit vector $h \in E \ominus F$, we have

$$
\operatorname{tr}\left(f\left|h^{*}\right|^{2}\right)=\langle f h, h\rangle=0, \quad f \in J,
$$

since $f h \in J E \subset F$. Since $A=J+\mathbb{C} 1$ it follows that $\operatorname{tr}\left(\cdot\left|h^{*}\right|^{2}\right)$ is a normal state on $M$ extending $\varphi$.

Remark 2.3. An alternative quick (and more noncommutative) proof which also relies on the $A=J+\mathbb{C} 1$ relation: Suppose that $M$ acts on a Hilbert space $K$, so that $A \subset B(K)$. Consider the amplification $\pi(x)=x^{(\infty)}$ of the identity representation of $M$, acting on the countably infinite direct sum $K^{(\infty)}$ of copies of $K$. Then $\pi(J)$ 
is reflexive by e.g. [5. Appendix A.1.5], and by definition of reflexive there, since $I \notin \pi(J)$, there is a vector $\xi \in K^{(\infty)}$ with $\xi \notin[\pi(J) \xi]$. Hence $[\pi(A) \xi] \ominus[\pi(J) \xi] \neq(0)$. Choose $\eta \in[\pi(A) \xi] \ominus[\pi(J) \xi]$ of norm 1 . Since $\pi(J) \eta \in \pi(J)[\pi(A) \xi] \subset[\pi(J) \xi]$ it follows that $\langle\pi(\cdot) \eta, \eta\rangle$ is a normal state on $M$ annihilating $J$. Hence its restriction to $A$ is a multiple of $\Phi$, indeed equals $\varphi$ since both are states.

Theorem 2.4. Consider the inclusions $D \subset A \subset M$, where $M$ is a von Neumann algebra, $A$ is a weak ${ }^{*}$ closed subalgebra of $M$, and $D$ is an atomic von Neumann subalgebra containing the unit of $M$. If $\Phi: A \rightarrow D$ is a unital weak ${ }^{*}$ continuous homomorphism which is also a D-bimodule map, then $\Phi$ extends to a normal conditional expectation $\Psi: M \rightarrow D$.

Proof. The case that $D=\mathbb{C} 1$ follows immediately from Theorem 2.2.

Next suppose that $D$ is a type I factor, thus isomorphic to $B\left(l^{2}(I)\right)$ for an index set $I$. We may suppose that $M=B(K)$, and that $D$ is the range of a normal unital *-homomorphism $\pi: B\left(l^{2}(I)\right) \rightarrow B(K)$. For $i \in I$ let $p_{i}=\pi\left(E_{i i}\right)$, where $\left\{E_{i k}\right\}$ are the matrix units in $B\left(l^{2}(I)\right)$, and set $L=p_{j} K$ for a fixed $j \in I$. By a matrix unit argument we may suppose that (unitarily) $K=L^{(I)}=L \otimes l^{2}(I)$, the Hilbert space sum of $I$ copies of $L$, that $M=B(L) \bar{\otimes} B\left(l^{2}(I)\right)$, and $D=I_{L} \otimes B\left(l^{2}(I)\right)$ and $p_{i}=I_{L} \otimes E_{i i} \in D \subset A$. Set $A_{j}=p_{j} A p_{j}$ and

$$
B=\left\{T \in B(L): p_{j}(T \otimes I) p_{j} \in A_{j} \subset A\right\} .
$$

Then by a routine matrix unit argument we have $B \cong B \otimes E_{j j}=A_{j}$ and $A=$ $B \bar{\otimes} B\left(l^{2}(I)\right)$ via the unitary above. Here $B \bar{\otimes} B\left(l^{2}(I)\right)$ is the normal spatial tensor product of e.g. 2.7.5 (2) in [5], namely the weak* closure of $B \otimes B\left(l^{2}(I)\right)$ in the von Neumann algebra $B(L) \bar{\otimes} B\left(l^{2}(I)\right) \cong B(K)$. That $A=B \bar{\otimes} B\left(l^{2}(I)\right)$ follows by a matrix unit argument (exploiting the usual properties of matrix units like $\left.E_{i k}=E_{i j} E_{j k}=E_{i j} E_{j j} E_{j k}\right)$ and weak* density. One also uses the fact that $A$ contains, and is a submodule over, $D=I_{L} \otimes B\left(l^{2}(I)\right)$, that $A_{j}=B \otimes E_{j j}$, and that the span of the $p_{i} A p_{k}$ is weak* dense in $A$.

For $i, k \in I, \Phi\left(p_{i} a p_{k}\right)=p_{i} \Phi(a) p_{k} \in \mathbb{C} E_{i k}$. Hence $\Phi\left(b \otimes E_{i k}\right)$, viewed as a matrix in $B\left(l^{2}(I)\right)$, is zero except perhaps for its $i$ - $k$ entry, for any $b \in B$. Let $\varphi=\pi_{j j} \circ \Phi \circ \epsilon_{j}$, where $\epsilon_{j}(b)=b \otimes E_{j j}$ and $\pi_{j j}$ is the state on $D$ that evaluates the $j$ - $j$ entry. Then $\varphi$ is a character of $B$. Also, $\Phi=\varphi \otimes I$, indeed for $b \in B$ and $i, k \in I$ we have

$$
\Phi\left(b \otimes E_{i k}\right)=E_{i j} \Phi\left(b \otimes E_{j j}\right) E_{j k}=E_{i j} \varphi(b) E_{j k}=(\varphi \otimes I)\left(b \otimes E_{i k}\right) .
$$

first paragraph, $\varphi$ extends to a normal state $\sigma$ on $B(L)$, so that $\Psi=\sigma \otimes I$ is a normal UCP map extension of $\Phi$ to $M=B(L) \bar{\otimes} B\left(l^{2}(I)\right)$.

Finally, suppose that $D$ is atomic, so $D \cong \oplus_{i \in I} B\left(H_{i}\right)$ for Hilbert spaces $H_{i}$. Let $p_{i}$ be the central projection in $D$ corresponding to the identity in $B\left(H_{i}\right)$. We have that $\Phi\left(p_{i} a p_{j}\right)=p_{i} \Phi(a) p_{j}=0$ for $i \neq j$ (since $p_{i}$ is central in $D$ ). Thus $\Phi=\Phi \circ \Delta_{\mid M}$ where $\Delta$ is the UCP map on $M$ defined by $\Delta(x)=\sum_{i} p_{i} x p_{i}$. Let $\Phi_{i}=\Phi_{\mid p_{i} A p_{i}}$. By the last paragraph $\Phi_{i}$ extends to a normal UCP map $\sigma_{i}: p_{i} M p_{i} \rightarrow p_{i} D p_{i}$. We obtain a normal UCP map extension $\Psi=\left(\oplus_{i} \sigma_{i}\right) \circ \Delta$ of $\Phi$ to $M$. By Tomiyama's well known theorem (see p. 132-133 in 1]) on projections of norm 1, $\Psi$ is necessarily a $D$-bimodule map and conditional expectation.

Proposition 2.5. Suppose that $A$ is a unital weak ${ }^{*}$ closed subalgebra of $M=B(H)$, and suppose that $D$ is a weak* closed unital selfadjoint subalgebra of $A$. Suppose that 
$\Phi: A \rightarrow D$ is a weak ${ }^{*}$ continuous unital homomorphism on $A$ that is a D-bimodule map. Suppose that there exists a normal positive map $\Psi: M \rightarrow D$ extending $\Phi$. Then $D$ is an atomic von Neumann algebra.

Proof. Note that such a map $\Psi$ is necessarily a normal norm 1 projection onto $D$. However a von Neumann algebra which is the range of such a projection on $B(H)$ is atomic ([1, Theorem IV.2.2.2]).

The following example shows the importance of the selfadjointness of $D$ in finding any positive $B(H)$-valued extension of a homomorphism.

Proposition 2.6. There exists inclusions $D \subset A \subset M$, where $M$ is a finite von Neumann algebra, and $A$ and $D$ are commutative finite dimensional weak* closed subalgebras of $M$ containing the unit of $M$, with the following properties.

(1) The subalgebra $D$ may be identified (completely isometrically) with a unital subalgebra $D$ of the $2 \times 2$ matrices $M_{2}$.

(2) There exists a weak* continuous completely contractive unital homomorphism $\Phi: A \rightarrow D \subset M_{2}$, such that $\Phi$ has no contractive or positive weak* continuous linear extension from $M$ to $M_{2}$ or to $D$.

(3) If $\Phi$ is viewed as a map $A \rightarrow D \subset A$ then $\Phi$ is an idempotent $D$-bimodule map.

Proof. Let $\varphi_{1}$ be the weak* continuous state on $\mathcal{S} \subset L^{\infty}([0,1])$ in Example 2.1] Let $M=M_{2}\left(L^{\infty}([0,1])\right)$, the $W^{*}$-algebra of $2 \times 2$ matrices with entries in $L^{\infty}([0,1])$. Let $A$ be the subalgebra of $M_{2}\left(L^{\infty}([0,1])\right)$ consisting of upper triangular matrices with scalars (constant functions) as the main diagonal entries and elements from $\mathcal{S}$ in the 1-2 entry. This $A$ is four dimensional. It is also weak* closed in $M$ since any finite dimensional subspace is closed in any linear topology. This is related to [5. Lemma 2.7.7]. Define $\Phi: A \rightarrow M_{2}$ to be the map that applies $\varphi_{1}$ in the 1-2 entry, and leaves other entries 'unchanged'. That is $\Phi$ is 'evaluation' at 1 . This is easily seen to be a weak* continuous unital homomorphism. Also, $\Phi$ is (completely) contractive by [5. Proposition 2.2.11]. Let $D$ be the range of $\Phi$, the upper triangular $2 \times 2$ matrices. We may also view $D \subset A$ by identifying an upper triangular matrix with the same matrix in $A$, but with 1-2 entry multiplied by the monomial $t$. Then $\Phi$ viewed as a map $A \rightarrow D \subset A$ is an idempotent $D$-bimodule map. Suppose that $R: M \rightarrow M_{2}$ was a weak ${ }^{*}$ continuous contractive extension of $\Phi$. Then $R$ is positive (since it is well known that contractive unital maps on $C^{*}$-algebras are positive). The restriction of $R$ to matrices that are only nonzero in their 1-2 entry, followed by the projection onto the 1-2 entry, defines a weak* continuous contractive functional $\psi$ on $L^{\infty}([0,1])$. Finally, it is clear that $\psi$ extends $\varphi_{1}$, contradicting the first paragraph of this section. This contradiction shows that our extension $R$ cannot be positive or contractive.

One may adjust $A$ in the proof above to be three dimensional by taking the main diagonal entries of matrices in $A$ to be equal. We also remark that if one insists on bimodule map extensions then one may get counterexamples with $M$ finite dimensional (see e.g. [11, Example 3.5]).

We also remark that very strong forms of the noncommutative Hoffman-Rossi theorem hold for Arveson's maximal subdiagonal subalgebras of $\sigma$-finite von Neumann algebras 3 . (or more generally for algebras having some of the GleasonWhitney properties GW1, GW2, GW from the start of Section 4 in [2, or their 
variants for states, and studied further in e.g. [3, Section 5] in the $\sigma$-finite case). See Section 3 of [4] for more on this.

Acknowledgment. Noncommutative Hoffman-Rossi theorems were a project suggested (and guided in its more technical parts, e.g. things involving von Neumann algebras) by the first author for the second author's M. S. thesis [7] supervised by the third author. The present paper contains several advances made subsequent to that reference, including the main result. We also thank the referee for several comments.

\section{REFERENCES}

[1] B. Blackadar, Operator algebras. Theory of $C^{*}$-algebras and von Neumann algebras, Encyclopaedia of Mathematical Sciences, 122. Operator Algebras and Non-commutative Geometry, III. Springer-Verlag, Berlin, 2006.

[2] D. P. Blecher and L. E. Labuschagne, Noncommutative function theory and unique extensions, Studia Math. 178 (2007), 177-195.

[3] D. P. Blecher and L. E. Labuschagne, Ueda's peak set theorem for general von Neumann algebras, Trans. Amer. Math. Soc. 370 (2018), 8215-8236.

[4] D. P. Blecher and L. E. Labuschagne, On vector-valued characters for noncommutative function algebras, preprint (2019).

[5] D. P. Blecher and C. Le Merdy, Operator algebras and their modules-an operator space approach, Oxford Univ. Press, Oxford (2004).

[6] G. Cassier, Algébres duales uniformes d'opérateurs sur l'espace de Hilbert, Studia Math. 95 (1989), 17-32.

[7] L. C. Flores, Non-commutative weak*-continuous operator extensions, M.S. thesis, Texas A\& M University-Corpus Christi, May 2018.

[8] T. W. Gamelin, Uniform Algebras, Second edition, Chelsea, New York, 1984.

[9] K. Hoffman and H. Rossi, Extensions of positive weak*-continuous functionals, Duke Math. J. 34 (1967), 453-466.

[10] H. König, Zur abstrakten theorie der analytischen funktionen, Math. Z. 88 (1965), 136-165.

[11] R. R. Smith, Completely bounded module maps and the Haagerup tensor product, J. Funct. Anal. 102 (1991), 156-175.

[12] M. Takesaki, Conditional expectations in von Neumann algebras, J. Functional Analysis 9 (1972), 306-321.

Department of Mathematics, University of Houston, Houston, TX 77204-3008, USA

E-mail address, David Blecher: dblecher@math.uh.edu

Department of Mathematics, University of Missouri, Columbia, MO 765212, USA

E-mail address, Luis Flores: lcfvd6@mail.missouri.edu

Department of Mathematics and Statistics, Texas A \& M University-Corpus Christi, Corpus Christi, TX 78412-5825, USA

E-mail address, Beate Zimmer: beate.zimmer@tamucc.edu 